\title{
Asociación entre el consumo de alcohol y la infección por virus de inmunodeficiencia humana
}

\author{
Omayra Chincha L., Frine Samalvides C., Antonio Bernabé-Ortiz, Hever Kruger P. y Eduardo Gotuzzo H.
}

\section{Association between alcohol consumption and human immunodeficiency virus infection}

Several factors have been associated with HIV infection, but alcohol consumption has not been studied completely. The purpose of this study is to evaluate the association between alcohol consumption and HIV infection. A case-control study was undertaken with HIV-positive and HIV-negative patients, matched by sex and age ( \pm 2 years). Latin-American Alcoholism Test (long version) was used to evaluate alcoholism diagnosis. A total of 198 cases were matched with 198 controls: 38.9\% of cases were diagnosed as alcoholics, and only $22.7 \%$ of controls ( $<<0.001)$. The multivariate model showed that economic income $(p<0.001)$, sexual orientation ( $p<0.001)$, and alcoholism diagnosis $(p<0.001)$ were associated with HIV infection. This study demonstrates an association between alcohol consumption, homosexual practices between men and economic income with the prevalence of HIV infection.

Key words: HIV infection, alcoholism, homosexuality, case-control study.

Palabras clave: VIH, alcoholismo, homosexualidad, casos y controles.

\section{Introducción}

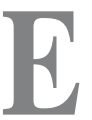
l Instituto Nacional de Abuso de Alcohol y Alcoholismo (NIAAA) de los Estados Unidos de América reporta que las personas con desórdenes de consumo de alcohol tienen mayor probabilidad de contraer infección por VIH y otras enfermedades de transmisión sexual que la población general ${ }^{1}$, debido a su asociación con conductas sexuales de riesgo, tales como múltiples parejas sexuales, relaciones sexuales sin protección, relaciones con trabajadoras sexuales y sexo a cambio de alcohol o drogas ${ }^{2}$.

Asimismo, personas con la infección por VIH son más propensas a consumir mayores cantidades de alcohol $^{1}$, lo que puede dificultar la adherencia para la terapia anti-retroviral ${ }^{3,4}$.

Diversas sustancias, entre ellas el alcohol, pueden actuar como modificadores de la conducta sexual, haciendo que los consumidores tengan mayores niveles de relaciones sexuales no protegidas muy próximas al uso de la sustancia ${ }^{5,6}$.

Siendo en nuestro país la relación entre el consumo de alcohol y la infección por VIH aún poco conocida, se realiza el presente estudio con el propósito de evaluar la asociación entre el consumo de alcohol y la infección por VIH, buscando ampliar los conocimientos acerca de un factor asociado importante para la transmisión.

\section{Pacientes y Métodos}

Diseño. Estudio observacional analítico, de tipo caso-control, empleando casos de infección por VIH, reportados en el Departamento de Enfermedades Infecciosas, Tropicales y Dermatológicas del Hospital Nacional Cayetano Heredia (Lima, Perú), durante el lapso entre agosto del 2003 y diciembre del 2004, de manera constante e ininterrumpida.

Tamaño de muestra. Para el cálculo del tamaño de muestra se consideró que la proporción de consumo de alcohol en la población no infectada por VIH en Perú fue de $21 \%^{7}$ y que la proporción del consumo de alcohol en el grupo infectado por VIH fue del $35 \% \%^{1,8}$. Además, utilizando un nivel de confianza del $95 \%$ y un poder del $80 \%$, se obtuvo el número de muestras de 197 casos y 197 controles.

Criterios de selección. Los pacientes fueron enrolados de consultorios externos y del servicio de hospitalización. Los casos cumplieron los siguientes criterios de selección: pacientes entre 18 y 60 años de edad, con infección por VIH diagnosticada por ELISA y confirmada por Western Blot o Inmunofluorescencia Indirecta (IFI). Por otro lado, los controles fueron todos los pacientes de consultorios externos y del servicio de hospitalización, entre 18 y 60 años que acudie-
Universidad Peruana Cayetano Heredia Lima, Perú (OCH, HKP) Instituto de Medicina Tropical Alexander Von Humboldt Hospital Nacional Cayetano Heredia Departamento de Enfermedades Infecciosas, Tropicales y Dermatológicas (FSC, EGH) Facultad de Salud Pública y Administración

Unidad de Epidemiología, ETS y VIH/ SIDA (ABO)

Recibido: 1 de marzo de 2007 Aceptado: 28 de diciembre de 2007

\section{Correspondencia a:} Omayra Chincha Lino omychin@hotmail.com 
Después de leer cuidadosamente cada pregunta y según crea conveniente, marque con una cruz el espacio correspondiente a SI o NO. En ocasiones, usted podrá tener la impresión de que una o varias oraciones no están en relación directa con su problema; ante esta situación, elija de todas maneras una de las posibilidades. Por ejemplo, en caso de que usted nunca haya ingerido bebidas etílicas, entonces en cada una de las 13 preguntas Usted deberá marcar con una cruz el espacio que corresponde al no. Por el contrario, si usted sí ha llegado a consumir bebidas alcohólicas y además ya ha intentado, por algún tiempo, vivir sin tomar, entonces usted deberá marcar con una cruz el espacio que corresponde al sí en la quinta oración.

1. Si no tomara, tendría menos problemas $\quad \mathrm{NO}$

2. Pienso que debería tomar menos $\quad \mathrm{NI}$

3. Pienso que el beber está destruyendo mi vida $\quad \mathrm{NO}$

4. Si no tomara, me sentiría mejor conmigo mismo

5. Ya he intentado, por algún tiempo, vivir sin tomar SI NO

6. Después de tomar, tengo remordimientos o sentimientos de culpa $\quad$ NO

7. Me dan ganas de dejar de beber, pero luego vienen los deseos de seguir tomando SI NO

8. Después de tomar la primera copa, irremediablemente me dan ganas de seguir bebiendo SI NO

9. Si no tomara, no tendría problemas con mi pareja (esposa(o) o con mi novia(o)) o enamorada(o) SI NO

10. Actualmente me siento amargado por los problemas que ha ocasionado mi forma de beber

11. Para mujeres. consumo una o varias veces al mes más de 6 litros de cerveza o 3 litros (4 botellas) de vino o 1/4 partes de una botella de cualquier tipo de bebidas destiladas: aguardiente, ron, brandy, whisky, ginebra, vodka, tequila, mezcal

SI NO

Para hombres. consumo una o varias veces al mes más de 7 litros de cerveza o 4 litros ( 5 botellas) de vino o 1 botella de cualquier tipo de bebidas destiladas: aguardiente, ron, brandy, whisky, ginebra, vodka, tequila, mezcal

12. Las demás personas no entienden por qué tomo

13. Ya he empleado sistemas para beber menos, como ejemplo, tomar sólo a determinadas horas del día

ron por otra enfermedad, seronegativos para la infección por VIH. Se excluyeron aquellos participantes con antecedente de consumo de drogas, enfermedad psiquiátrica o con discapacidad intelectual para decidir, así como los que no firmaron el consentimiento informado. A cada potencial participante se le entregó el consentimiento informado para lectura. Luego de aceptada la participación en el estudio y firmado el documento, se procedió a realizar el cuestionario. Finalizados los mismos, se colocaba la ficha con todos los datos en un sobre cerrado hasta el proceso de digitación y análisis.

Materiales. Se utilizó un cuestionario compuesto de tres partes: datos demográficos, datos de conducta sexual, y la Prueba Diagnóstica de Alcoholismo Latinoamericana Versión Larga (PDAL-VL) (Anexo 1). Por cada una de las 13 preguntas de la prueba sobre alcoholismo se obtenía un puntaje igual a uno (1) si la respuesta era afirmativa y cero (0), por respuesta negativa9; construyéndose de esta manera una escala de diagnóstico utilizada en este estudio: no alcoholismo (0-4), sospecha de alcoholismo (5-7) y alcoholismo (8-13).

Análisis estadístico. Se creó una base de datos en una hoja de cálculo de Microsoft Excel, la cual fue trasladada para el análisis estadístico especializado a SPSS 11.0 (SPSS Inc, Chicago IL, E.U.A). La prueba $\chi^{2}$ fue utilizada para evaluar la asociación de variables categóricas, incluido el consumo de alcohol, con la infección por VIH. Posteriormente, se procedió al cálculo univariado de los Odd Ratios (OR) a través del análisis de regresión logística condicional (ajustado por edad y sexo). Con aquellas variables que mostraron asociación ( $\mathrm{p}<0,10)$ se construyó una serie de modelos multivariados, de los cuales se escogió el mejor de acuerdo al Likelihood Ratio, tomando un $\mathrm{p}<0,05$ como significativo y un intervalo de confianza (IC) a 95\%.

\section{Resultados}

De los 440 casos elegibles, 198 cumplían con los criterios de inclusión en el estudio y cada uno fue emparejado con un control del mismo sexo y edad \pm 2 años. La proporción de varones en ambos grupos fue de $62,1 \%$ mientras que la edad promedio fue de $34,2 \pm$ 9,5 años.

Variables sociodemográficas. Se encontró que el lugar de nacimiento en Lima $(p<0,01)$ y el ingreso económico menor a un salario mínimo vital* $(\mathrm{p}<0,001)$ estaban asociadas a la infección por VIH (Tabla 1).

Conductas sexuales. La orientación sexual ( $\mathrm{p}<0,001)$,

*salario mínimo vital $=500$ soles $=$ US 170 
las relaciones sexuales con personas recientemente conocidas $(\mathrm{p}<0,01)$ y la práctica de relaciones sexuales anales $(\mathrm{p}<0,001)$ estuvieron asociados a la infección por VIH (Tabla 2).

Consumo de alcohol. Evaluado por el resultado de los cuestionarios aplicados, el consumo de alcohol mostró asociación con la infección por VIH (p < 0,001) (Tabla 3).

El análisis bivariado demostró que el lugar de nacimiento, el ingreso económico, la orientación sexual, las relaciones sexuales con personas recientemente conocidas, la práctica de relaciones sexuales anales, así como el consumo de alcohol, fueron factores asociados.

El modelo multivariado final mostró que el ingreso económico $(\mathrm{p}<0,001)$, la orientación sexual $(\mathrm{p}<0,001)$ y el consumo de alcohol ( $\mathrm{p}<0,001)$ se comportaron como factores independientes asociados a la infección por VIH (Tabla 4).

\section{Discusión}

Este estudio demuestra una asociación entre el consumo de alcohol, la orientación homosexual y el ingreso económico menor a un salario mínimo vital, con la prevalencia de infección por VIH.

Varias de las prácticas sexuales entre hombres son factores de riesgo para adquisición de la infección por $\mathrm{VIH}^{10,11}$. Los hombres que tienen sexo con otros hombres (orientación bisexual u homosexual) tienen mayor número de parejas sexuales ${ }^{12}$, menor tasa de uso consistente de condones con sus parejas ${ }^{13,14}$ y mayor prevalencia de otras enfermedades de transmisión sexual que pueden potenciar el riesgo de adquisición del virus ${ }^{15,16}$. Más aún, todas estas conductas de riesgo, se ven reforzadas por el consumo de alcohol, especialmente en lo referente al uso de condón en las relaciones anales receptivas ${ }^{17}$.

La infección por VIH ha sido asociada a bajos niveles económicos así como a pobres niveles de educación $^{2,18,19}$. En este estudio, la asociación es más fuerte con el ingreso económico. La referencia usada en este caso es el sueldo mínimo vital, lo que nos permite también en forma indirecta evaluar el nivel de pobreza de la población seleccionada en el estudio. La pobreza, además de estar relacionada a un menor ingreso, se asocia a menor educación y menor conocimiento y uso de preservativos, así como a una reducida posibilidad de acceso a los servicios de salud, lo que podría incrementar el riesgo de infección por $\mathrm{VIH}^{20}$.
Tabla 1. Características sociodemográficas de la población e infección por VIH

\begin{tabular}{lrrrrr} 
Variable & \multicolumn{1}{c}{$\begin{array}{c}\text { Casos } \\
(\mathbf{n}=\mathbf{1 9 8})\end{array}$} & $\begin{array}{c}\text { Controles } \\
(\mathbf{n}=\mathbf{1 9 8})\end{array}$ & p \\
$\begin{array}{l}\text { Grado de instrucción } \\
\text { Ninguna-Primaria }\end{array}$ & 27 & $(13,6 \%)$ & 21 & $(10,6 \%)$ & 0,09 \\
$\begin{array}{l}\text { Secundaria } \\
\text { Técnico-Superior }\end{array}$ & 118 & $(59,6 \%)$ & 104 & $(52,5 \%)$ & \\
Lugar de nacimiento & 53 & $(26,8 \%)$ & 73 & $(36,9 \%)$ & \\
Lima & 140 & $(70,7 \%)$ & 118 & $(59,6 \%)$ & $<0,01$ \\
Fuera de Lima & 58 & $(29,3 \%)$ & 80 & $(40,4 \%)$ & \\
Ingreso económico & & & & & \\
Menor a 1 sueldo* & 87 & $(43,9 \%)$ & 54 & $(27,3 \%)$ & $<0,001$ \\
Igual a 1 sueldo & 73 & $(36,9 \%)$ & 71 & $(35,9 \%)$ & \\
Superior a 1 sueldo & 38 & $(19,2 \%)$ & 73 & $(36,9 \%)$ & \\
& & & & &
\end{tabular}

*Sueldo: ingreso económico igual a un sueldo mínimo vital.

Tabla 2. Conductas sexuales de la población en estudio e infección por VIH

Variable Casos controles p

\section{Orientación sexual}

Heterosexual

$(n=198)$

$(n=198)$

p

$\begin{array}{lrrrr}\text { Heterosexual } & 141 & (71,9 \%) & 180 & (93,8 \%) \\ \text { Bisexual } & 22 & (11,2 \%) & 10 & (5,2 \%) \\ \text { Homosexual } & 33 & (16,8 \%) & 2 & (1,0 \%)\end{array}$

Relaciones sexuales con personas recientemente conocidas

$\begin{array}{lllrrr}\text { No } & 21 & (61,1 \%) & 149 & (75,3 \%) & <0,01 \\ \text { Sí } & 77 & (38,9 \%) & 49 & (24,7 \%) & \end{array}$

Prácticas sexuales anales

$\begin{array}{lrrrrr}\text { No } & 147 & (74,2 \%) & 181 & (91,1 \%) & <0,001 \\ \text { Si } & 51 & (25,8 \%) & 17 & (8,9 \%) & \end{array}$

Relaciones sexuales con violencia*

$\begin{array}{llllll}\text { No } & 179 & (90,4 \%) & 191 & (96,5 \%) & 0,05\end{array}$

Sí

Relaciones sexuales grupales ${ }^{\#}$

$\begin{array}{lrrrrr}\text { No } & 189 & (95,5 \%) & 196 & (99,0 \%) & 0,05 \\ \text { Sí } & 9 & (4,5 \%) & 2 & (1,0 \%) & \end{array}$

* Relaciones sexuales en contra de la decisión de la persona

"Relaciones sexuales con más de una persona al mismo tiempo

Tabla 3. Asociación entre el consumo de alcohol y la infección por VIH

\begin{tabular}{llllll} 
Variable & $\begin{array}{c}\text { Casos } \\
(\mathbf{n}=\mathbf{1 9 8})\end{array}$ & \multicolumn{2}{c}{$\begin{array}{c}\text { Controles } \\
(\mathbf{n}=\mathbf{1 9 8})\end{array}$} & p \\
Consumo de alcohol & 83 & $(41,9 \%)$ & 120 & $(60,6 \%)$ & $<$ \\
No alcohólico & 38 & $(19,2 \%)$ & 33 & $(16,7 \%)$ & 0,001 \\
Sospecha de alcoholismo & 77 & $(38,9 \%)$ & 45 & $(22,7 \%)$ &
\end{tabular}


Tabla 4. Características de procedencia, ingreso económico, conducta sexual y consumo de alcohol en pacientes con infección por VIH. Análisis univariado y multivariado

$\begin{array}{lcc}\text { Variables } & \begin{array}{c}\text { Análisis univariado } \\ \text { HR (95\% IC) }\end{array} & \begin{array}{c}\text { Análisis multivariado } \\ \text { HR (95\% IC) }\end{array} \\ \begin{array}{l}\text { Lugar de nacimiento } \\ \text { Lima }\end{array} & 1 & \\ \text { Fuera de Lima } & 0.61(0,40-0,93) & 1 \\ \begin{array}{l}\text { Ingreso económico } \\ \text { Menor a } 1 \text { sueldo }\end{array} & 1 & 0,41(0,24-0,70) \\ \text { lgual a } 1 \text { sueldo } & 0,64(0,39-1,02) & 0,23(0,13-0,42) \\ \begin{array}{l}\text { Superior a } 1 \text { sueldo } \\ \text { Orientación sexual }\end{array} & 0,32(0,19-0,54) & 1 \\ \begin{array}{l}\text { Heterosexual } \\ \text { Bisexual }\end{array} & 1 & 2,79(1,9-6,51) \\ \text { Homosexual } & 2,81(1,29-6,12) & 30.87(7,03-135,57)\end{array}$

Relaciones sexuales con personas recientemente conocidas

No

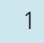

Sí

Relaciones sexuales anales

No

$1,94(1,26-2,98)$

Sí $\quad 3,53(1,95-6,38)$

Relaciones sexuales con violencia

No 1

Sí $2,90(1,19-7,05)$

Relaciones sexuales en grupales

No

Sí

\section{Consumo de alcohol}

Sin alcoholismo

Sospechoso de alcoholismo

Alcoholismo

$$
4,67(0,99-21,88)
$$ \\ $4,67(0,99-21,88)$}

1

$1,66(0,97-2,87)$

$2,47(1,56-3,93)$
1

$1,77(0,96-3,27)$

$2,75(1,62-4,66)$
El consumo de alcohol, por si solo, fue un factor independiente asociado a la infección por VIH. Una revisión sistemática previa ha demostrado que el alcohol produce cambios conductuales que pueden favorecer la exposición de riesgo de las personas, tanto varones como mujeres, especialmente en su sexuali$\operatorname{dad}^{21}$. Inclusive, el consumo de alcohol puede potenciar el efecto de otras drogas como la cocaína o el crack, lo que puede potenciar las conductas de riesgo $^{22}$, y disminuir la práctica de sexo seguro en las personas de diversas orientaciones sexuales ${ }^{23}$. Asimismo, otros estudios han mostrado que la ingesta de grandes cantidades de alcohol está asociada a falla en la adherencia a tratamiento de largo plazo como es el caso del $\mathrm{VIH}^{4}$, por lo que es necesaria la aplicación de otras estrategias para evitar el desarrollo de formas resistentes del virus ${ }^{24}$.

Una ventaja de este estudio es que utiliza una encuesta validada para la medición del consumo de alcohol, (PDAL-VL), lo que permite el diagnóstico de alcoholismo y demuestra la asociación de este factor de riesgo con la infección por VIH. Si bien este cuestionario contiene más preguntas que otros previamente validados $^{25}$, fue útil en la evaluación del consumo de alcohol previo ${ }^{9}$.

La limitación del estudio es que no permite interpretar en forma irrefutable si el alcoholismo es un factor de riesgo para adquirir el $\mathrm{VIH}^{26}$. El consumo de alcohol puede estar asociado a pobreza, menor nivel educativo, marginalidad, etc, factores que también están relacionados a la infección por el VIH por el poco conocimiento de la enfermedad y menor acceso a los servicios de salud. Además, el consumo de alcohol puede ser consecuencia del daño neurológico propio de la encefalopatía por VIH, que predispone a un mayor consumo de alcohol ${ }^{27}$ o por trastornos psiquiátricos como la depresión. A su vez, la infección por VIH puede ser secundaria al consumo de alcohol, por los comportamientos sexuales inadecuados causados por el consumo ${ }^{11}$. Sin embargo, los resultados nos permiten concluir que la ingesta de alcohol es un factor independiente asociado a la infección por VIH.

Debido a que excluimos del estudio a los pacientes usuarios de drogas, no pudimos evaluar el efecto de esta variable en el estudio, ni la relación que podría existir de la combinación consumo de alcohol y drogas, lo cual puede producir un sesgo en el estudio. No obstante, la prevalencia de consumo de drogas es baja en nuestro medio y, más aún, sólo alrededor de 0,5\% de los casos de infección por VIH en el Perú se explican por el uso de drogas intravenosas, por lo que se hubiese necesitado un mayor número de pacientes para detectar dicho efecto.

Con todo, el problema de alcoholismo debe ser estudiado en cada paciente seropositivo, efectuando un enfoque integral del problema y la búsqueda de soluciones terapéuticas con un abordaje multidisciplinario de la enfermedad.

En conclusión, las intervenciones de salud pública no sólo deben centrarse en medidas reconocidas que disminuyen la transmisión de la infección por VIH, sino también en el diagnóstico de alcoholismo, ya que el abuso de alcohol puede llevar a prácticas sexuales de riesgo que incrementen la adquisición y transmisión del virus. En el enfoque terapéutico integral y multidisciplinario de un paciente afectado por la infección con VIH/SIDA, el alcoholismo debe ser abordado pues, al igual que otras dependencias, podría traer problemas de adherencia a tratamiento anti-retroviral. 


\section{Resumen}

Se conocen diversos factores asociados a la infección por VIH, siendo uno de los menos estudiados el consumo de alcohol. El propósito de este trabajo es evaluar la asociación entre el consumo de alcohol y la infección por VIH. Se realizó un estudio caso-control, pacientes con y sin infección por $\mathrm{VIH}$, apareados por sexo y edad ( \pm 2 años). Se utilizó la Prueba de Alcoholismo Latinoamericano Versión Larga para el diagnósti- co de alcoholismo. Se evaluaron 198 casos y 198 controles: $38,9 \%$ de los casos fueron diagnosticados de alcoholismo y sólo $22,7 \%$ de los controles ( $p<0,001$ ). El modelo multivariado mostró que el ingreso económico ( $p<0,001)$, la orientación sexual $(\mathrm{p}<0,001)$, así como el diagnóstico de alcoholismo ( $\mathrm{p}<0,001)$ se asociaron a la infección. Este estudio demuestra una asociación entre el consumo de alcohol, las prácticas homosexuales entre hombres y el ingreso económico con la prevalencia de infección por VIH.

\section{Referencias}

1.- Alcohol and HIV/AIDS. In: Alcohol Alert. Rockville: National Institute on Alcohol Abuse and Alcoholism; 2002. http:// pubs.niaaa.nih.gov/publications/aa57.html (Accedido enero 2004).

2.- de Oliveira L C, Pereira R G, Reis U C. Prevalence of human immunodeficiency virus infection in alcoholics. Mem Inst Oswaldo Cruz 2001; 96: 21-3.

3.- Kresina T F, Flexner C W, Sinclair J, Correia M A, Stapleton J T, Adeniyi-Jones S, et al. Alcohol use and HIV pharmacotherapy. AIDS Res Hum Retroviruses 2002; 18: 757-70.

4.- Samet J H, Horton N J, Meli S, Freedberg K A, Palepu A. Alcohol consumption and antiretroviral adherence among HIV-infected persons with alcohol problems. Alcohol Clin Exp Res 2004; 28: 572-7.

5.- Hendershot C S, George W H. Alcohol and sexuality research in the AIDS era: Trends in publication activity, target populations and research design. AIDS Behav 2007; 11: 217-26.

6.- Malow R M, Devieux J G, Rosenberg R, Samuels D M, Jean-Gilles M M. Alcohol use severity and HIV sexual risk among juvenile offenders. Subst Use Misuse 2006; 41: 1769-88.

7.- Lucho M L, Jeri F R. Abuso y adicción a sustancias psicoactivas en pacientes infectados por el virus de inmunodeficiencia humana (VIH). Rev Neuro-Psiquiatr 2004; 67: 64-79.

8.- Molgaard C A, Nakamura C, Hovell M, Elder J P. Assessing alcoholism as a risk factor for acquired immunodeficiency syndrome (AIDS). Soc Sci Med 1988; 27: 1147-52.

9.- Gorenc K D, Peredo S, Ramírez J A, Abreu L F, Infante C, Oblitas L A, et al. Verdad de primer orden: La inconmensurabilidad del alcoholismo. Los errores en la medición de la Prueba Diagnóstica de Alcoholismo Latinoamericana (PDAL) a través de la simulación baudrillardiana contrainducida. Revista de
Neuro-Psiquiatría del Perú 1998; LXI(1).

10.- Shaffer D N, Njeri R, Justice A C, Odero W W, Tierney W M. Alcohol abbuse among patients with and without HIV infection attending public clinics in western Kenya. East Afr Med J 2004; 81: 594-8.

11.- Zablotska I B, Gray R H, Serwadda D, Nalugoda F, Kigozi G, Sewankambo N, et al. Alcohol use before sex and HIV acquisition: a longitudinal study in Rakai, Uganda. AIDS 2006; 20: 1191-6.

12.- Tabet S, Sánchez J, Lama J, Goicochea P, Campos P, Rouillon M, et al. HIV, syphilis and heterosexual bridging among Peruvian men who have sex with men. AIDS 2002; 16: $1271-7$.

13.- Renzi C, Tabet S R, Stucky J A, Eaton N, Coletti AS, Surawicz C M, et al. Safety and acceptability of the Reality condom for anal sex among men who have sex with men. AIDS 2003; 17: 727-31.

14.- Sanchez J, Gotuzzo E, Escamilla J, Carrillo C, Phillips I A, Barrios C, et al. Gender differences in sexual practices and sexually transmitted infections among adults in Lima, Peru. Am J Public Health 1996; 86: 1098-107.

15.- Pisani E, Girault P, Gultom M, Sukartini N, Kumalawati J, Jazan S, et al. HIV, syphilis infection, and sexual practices among transgenders, male sex workers, and other men who have sex with men in Jakarta, Indonesia. Sex Transm Infect 2004; 80: 536-40.

16.- Renzi C, Douglas J M Jr, Foster M, Critchlow C W, Ashley-Morrow R, Buchbinder SP, et al. Herpes simplex virus type 2 infection as a risk factor for human immunodeficiency virus acquisition in men who have sex with men. J Infect Dis 2003; 187: $19-25$.

17.- Irwin T W, Morgenstern J, Parsons J T, Wainberg M, Labouvie E. Alcohol and sexual HIV risk behavior among problem drinking men who have sex with men: An event level analysis of timeline follow back data. AIDS Behav 2006; 10: 299-307.

18.- Boily M C, Godin G, Hogben M, Sherr L, Bastos F I. The impact of the transmission dynamics of the HIV/AIDS epidemic on sexual behaviour: a new hypothesis to explain recent increases in risk takingbehaviour among men who have sex with men. Med Hypotheses 2005; 65: 215-26.

19.- Caceres C, Konda K, Pecheny M, Chatterjee A, Lyerla R. Estimating the number of men who have sex with men in low and middle income countries. Sex Transm Infect 2006; 82 Suppl 3:iii3-9.

20.- Peragallo N, Deforge B, O'Campo P, Lee S M, Kim Y J, Cianelli R, et al. A randomized clinical trial of an HIV-riskreduction intervention among low-income Latina women. Nurs Res 2005; 54: 108-18.

21.- Kalichman S C, Simbayi L C, Kaufman M, Cain D, Jooste S. Alcohol use and sexual risks for HIV/AIDS in Sub-Saharan Africa: Systematic review of empirical findings. Prev Sci 2007; 8: 141-51

22.- Krupitsky E M, Horton N J, Williams E C, Lioznov D, Kuznetsova M, Zvartau E, et al. Alcohol use and HIV risk behaviors among HIV-infected hospitalized patients in St. Petersburg, Russia. Drug Alcohol Depend 2005; 79: 251-6.

23.- Koblin B A, Husnik M J, Colfax G, Huang Y, Madison M, Mayer K, et al. Risk factors for HIV infection among men who have sex with men. AIDS 2006; 20: 731-9.

24.- Samet J H, Horton N J, Meli S, Dukes K, Tripps T, Sullivan L, et al. A randomized controlled trial to enhance antiretroviral therapy adherence in patients with a history of alcohol problems. Antivir Ther 2005; 10: 83-93.

25.- Samet J H, Phillips S J, Horton N J, Traphagen E T, Freedberg K A. Detecting alcohol problems in HIV-infected patients: use of the CAGE questionnaire. AIDS Res Hum Retroviruses 2004; 20: 151-5.

26.- Cook R L, Clark D B. Is there an association between alcohol consumption and sexually transmitted diseases? A systematic review. Sex Transm Dis 2005; 32: 156-64.

27.- Rothlind J C, Greenfield T M, Bruce A V, Meyerhoff D J, Flenniken D L, Lindgren J A, et al. Heavy alcohol consumption in individuals with HIV infection: effects on neuropsychological performance. J Int Neuropsychol Soc 2005; 11: 70-83. 